\title{
PM10 dispersion model in quarrying activities: a comparison of an ISC3 approach to a mono/multivariate geostatistical estimation
}

\author{
G. Alfaro Degan, G. Di Bona, D. Lippiello \& M. Pinzari \\ ${ }^{I}$ Dipartimento di Ingegneria Meccanica e Industriale, \\ Università degli studi ROMA TRE, Roma, Italy
}

\begin{abstract}
Airborne particulate matter represents one of the most pollutant factors in quarrying activities. The present study, developed in an Italian limestone quarry, pursues the aim of comparing different modelling and forecasting techniques for local atmospheric dispersion of PM10. In particular, after the preliminary characterization of dust sources according with an active monitoring system, dust concentration is sampled together with the most important environmental parameters such as pressure, temperature, atmospheric humidity, wind direction and speed. Then emission rate for each mining activity is determined. In particular typical mining activities are characterized with particular care of crushing, drilling, riddling, materials loading and unloading, transport and haul road. Therefore from this variety of sources associated with quarrying activities, the assessment of PM10 concentration is realized according with an ISC3 model. The second part of the work consists in the prediction of spatial variability of dust concentration according with a geostatistical approach. In particular, sampled values represent the input data grid used to obtain a continuous map of PM10 concentration in the quarry itself. For the monovariate approach, after a preliminary analysis based on variograms computation, the Kriging method is used. In the multivariate approach, taking into consideration the two variables PM10 and PTS, the Co-Kriging technique is used. In the final part a comparison of these two different methods is given in order to define the more suitable one in the exposed conditions.
\end{abstract}

Keywords: air pollution, particulate matter, dispersion, geostatistics. 


\section{Site selection}

The study was developed in a limestone quarry that sells crushed limestone for bituminous conglomerates (400-500 $\mathrm{m}^{3} /$ day). The flow chart of quarrying activities can be represented as it follows:

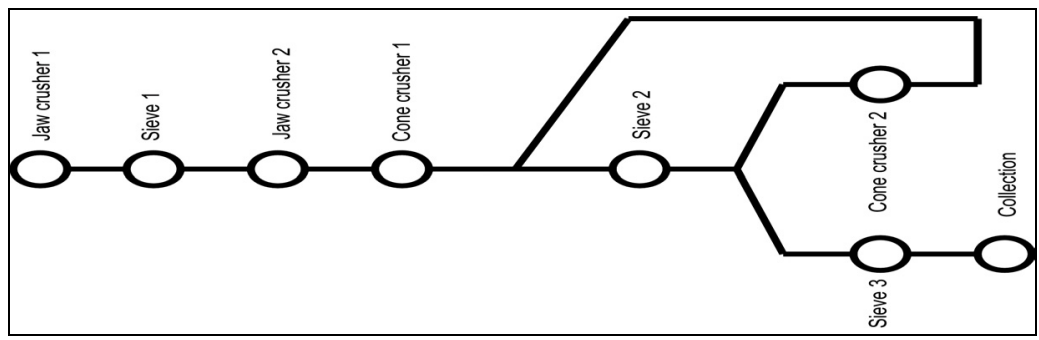

Figure 1: Quarrying activities: flow chart.

\section{Dust sources and their classification}

As for the exposed aim, the first step is represented by dust source individuation and classification. In particular, dust sources can be classified as ordinary or extraordinary sources, depending on the nature of activity that implies dust emission. They can be located in a specific position or distributed over a large area. Moreover, dust emission could last for the entire eight hour working day or just for a short period. In this classification it is important to find, according to a non-specific observation site, all the process activities connected with airborne dust emission independently from the intensity, which will be sampled afterwards. Also, in particular, we define point linear and area sources, but also uniform and not uniform emission sources.

\section{Dust source characterization: Upwind-downwind method}

This method allows one to calculate the amount of dust from a particular source. The dust concentrations upwind and downwind of the pollutant source are measured. The upwind sample is used for defining the degree of isolation of the source, while the downwind one is used to define the emission rate according to the application of the inverse formula of Gaussian Equation

\section{The ISC model}

The ISC3 is used for modeling dust dispersion in mining operations. It is based upon the Gaussian Equation for point source emissions

$$
C=\frac{Q K V D}{2 \pi u_{s} \sigma_{y} \sigma_{z}} \exp \left[-\frac{1}{2}\left(\frac{y}{\sigma_{y}}\right)^{2}\right]
$$


where

$\mathrm{Q}=$ pollutant emission rate $(\mathrm{g} / \mathrm{s})$

$\mathrm{K}=$ Conversion factor

$\mathrm{V}=$ Vertical term (include the images method)

$\mathrm{D}=$ Decay term

$\sigma_{y} \sigma_{z}=$ Standard deviation of lateral and vertical concentration distribution

$u_{s}=$ Mean wind speed at release high:

$\mathrm{C}=$ hourly concentration at downwind distance $\mathrm{x}$

$\mathrm{y}=$ crosswind distance from source to receptor

\section{$5 \quad$ Field studies}

\subsection{Crushers}

These sources are handled as volume sources as shown in the following figures.

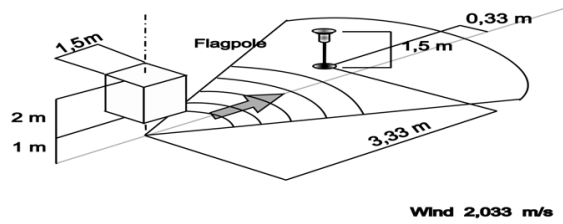

Figure 2: $\quad$ Volume source as handled by the ISC3 Model.

The results of the sampling procedure are summarized in the following table. The emission rate in order to obtain the sampled concentration in the sampling position is $40,77 \mathrm{mg} / \mathrm{s}$.

Table 1.

\begin{tabular}{|l|c|}
\hline Unsampled weight filter (1h) & $0,0585 \mathrm{~g}$ \\
\hline Sampled weight filter $(1 \mathrm{~h})$ & $0,0628 \mathrm{~g}$ \\
\hline Air sampled volume & $2,998 \mathrm{~m}^{3}$ \\
\hline Standard Volume & $2,966 \mathrm{~m}^{3}$ \\
\hline Temperature & $293,25^{\circ} \mathrm{K}$ \\
\hline Wind velocity & $2,033 \mathrm{~m} / \mathrm{s}$ \\
\hline Dust concentration & $1448,094 \mathrm{ug} / \mathrm{m}^{3}$ \\
\hline
\end{tabular}

\subsection{Conveyor belts}

In order to consider topographical features of the site we have considered adjacent volume sources at different heights. The sampling results are in the table, every single source is characterized by an emission rate of $2,21 \mathrm{mg} / \mathrm{s}$. 


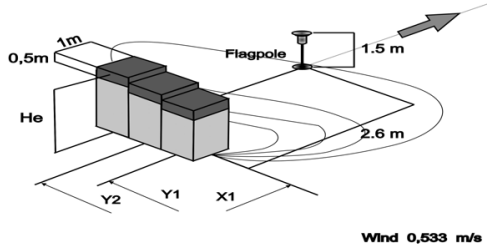

Figure 3: $\quad$ Line and uniform sources as handled by the ISC3 model.

The emission rate in order to obtain the sampled concentration $(1303,84$ $\mathrm{mg} / \mathrm{m}^{3}$ ) is defined according to the inverse Gaussian form of the equation.

Table 2.

\begin{tabular}{|l|c|}
\hline Unsampled weight filter (1h) & $0,0583 \mathrm{~g}$ \\
\hline Sampled weight filter $(1 \mathrm{~h})$ & $0,0615 \mathrm{~g}$ \\
\hline Sampling time. & $2,5 \mathrm{~h}$ \\
\hline Air sampled volume & $2,499 \mathrm{~m}^{3}$ \\
\hline Temperature & $299,15^{\circ} \mathrm{K}$ \\
\hline Wind velocity & $0,533 \mathrm{~m} / \mathrm{s}$ \\
\hline Dust concentration & $1303,849 \mathrm{ug} / \mathrm{m}^{3}$ \\
\hline
\end{tabular}

\subsection{Road dust emission}

A receptor to characterize the emission of haul trucks has been defined in order to estimate this source type. This application creates a constant uniform distribution of emissions over this specified area. In real life this source is not uniform, but it is handled like a uniform one to obtain the measured concentration.

A first sampling has been carried out with a watered area while the second one has been carried out with the real conditions.

Table 3.

\begin{tabular}{|c|c|}
\hline Dust mass & $0,0014 \mathrm{~g}$ \\
\hline Sampling time & $3 \mathrm{~h}$ \\
\hline Temperature & $300,81^{\circ} \mathrm{K}$ \\
\hline Wind speed & $2,700 \mathrm{~m} / \mathrm{s}$ \\
\hline Dust concentration & $482.91 \mathrm{ug} / \mathrm{m}^{3}$ \\
\hline
\end{tabular}

In case of area sources the rate emission is defined by surface unit $Q=0,729$ $\mathrm{mg} / \mathrm{s} \cdot \mathrm{m}^{2}$. 


\subsection{Two dimensional graph of dust concentration}

The field data of each source was input into the ISC3 model to obtain a two dimensional map of dust concentration in the quarry itself (1,7 $\mathrm{m}$ flagpole).
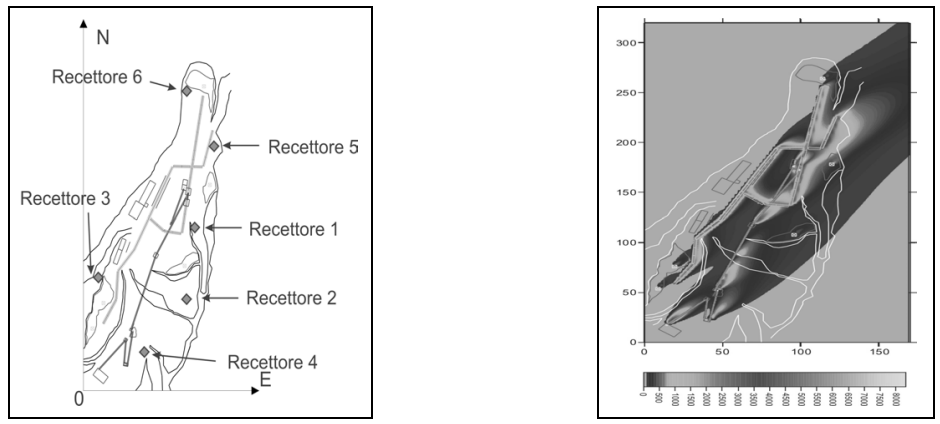

Figure 4: Receptors and complete simulation.

\section{Geostatistical application}

Geostatistics might be viewed as a methodology for interpolating data on an irregular pattern. Each data value is associated with a location in space and there is at least an implied connection between the location and the data value. It may be useful to introduce some geostatistical definitions for an exact interpretation of topics. In particular it is important to define:

\subsection{Variogram}

It is a geostatistical function used in characterizing the spatial variability of a local phenomenon.

Let $\mathrm{S}$ be a domain of definition of FA $\mathrm{z}(\mathrm{x})$ and given $\mathrm{x}_{0}$ and $\mathrm{x}_{0}+\mathrm{h}$ a couple of $\mathrm{S}$ points $\mathrm{h}$ distant.

$\left[z\left(x_{0}+h\right)-z\left(x_{0}\right)\right]$ is defined as a new variable called accretion, its half variance is for definition the variogram:

$$
\gamma\left(x_{0}, h\right)=1 / 2 \operatorname{Var}\left\{\left[z\left(x_{0}+h\right)-z\left(x_{0}\right)\right]\right\}
$$

The variogram estimation is realized according to experimental samples: it is shown the sampling points map on an irregular grid with different concentration values. As for stationary and semi-stationary functions, the accretion $\mathrm{z}(\mathrm{x}+\mathrm{h})$ $\mathrm{z}(\mathrm{x})$ does not change, so the value to be calculated will be:

$$
\gamma^{*}(h)=\sum_{i=1}^{N_{R}}\left[z\left(x_{i+r}\right)-z\left(x_{i}\right)\right]^{2}
$$

where: $\mathrm{N}$ represents the number of couples whose distance on $\mathrm{X}$ direction is $\mathrm{r}$. 

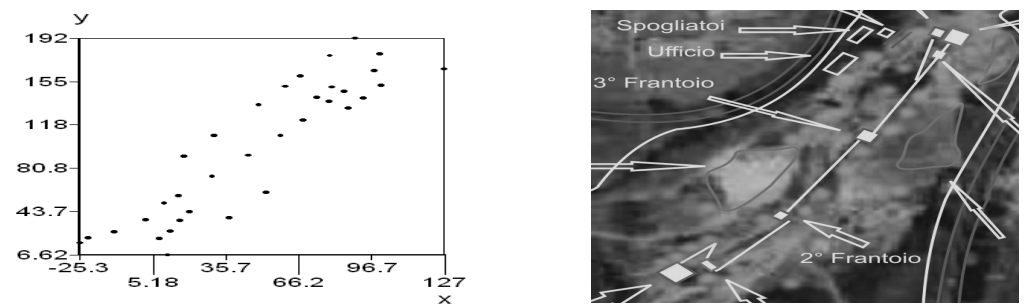

Figure 5: $\quad$ Input data grid and sampled points.

\subsection{Model of regionalization}

In order to create a variographic model, an experimental variogram of the $\mathrm{C}$ variable is realized. On such experimental evidence a model of two nested structures has been developed:

$>$ Hole component

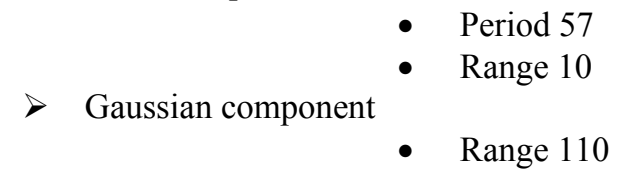

In order to fit the theoretical variogram with two nested structures only one direction $\left(0^{\circ}\right)$ has been considered because of the phenomenon's isotropy.

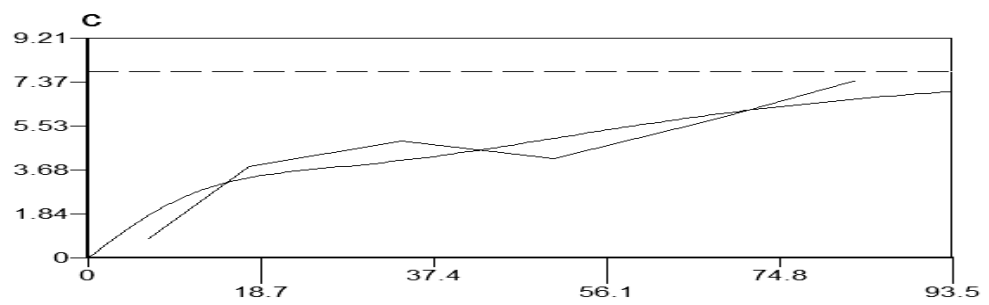

Figure 6: Variogram/model.

\subsection{Linear approach in geostatistical analysis: Ordinary Kriging}

$\mathrm{Z}(\mathrm{x})$ is an aleatory stationary function used to study the phenomenon in a probabilistic way, and $\mathrm{C}(\mathrm{h})$ and $\gamma(\mathrm{h})$ are covariance and variogram functions, respectively. $\mathrm{N}$ is the number of points required to estimate $\mathrm{x}_{0}, \mathrm{x}_{\alpha}$ and $\mathrm{z}\left(\mathrm{x}_{\alpha}\right)$ are with $\alpha=1, \mathrm{n}$ respectively their position and their corresponding variable values. $\mathrm{Z}\left(\mathrm{x}_{0}\right)$ is the unknown value in $\mathrm{x}_{0}, \mathrm{z}^{*}\left(\mathrm{x}_{0}\right)$ is the linear estimator considered. It is showed in the following form

$$
Z^{*}\left(X_{0}\right)=\sum_{\alpha=1}^{N} \lambda_{\alpha} Z\left(X_{\alpha}\right)
$$

where $\lambda_{\alpha}$ are the coefficients of a linear combination 
On this evaluation an associated estimation error, $\varepsilon$, is defined as the difference between the sampled value and the estimated value:

$$
\varepsilon=Z\left(X_{0}\right)-\sum_{\alpha=1}^{N} \lambda_{\alpha} Z\left(X_{\alpha}\right)
$$

However, it is also to be considered that

$$
\begin{gathered}
E\left[Z\left(X_{0}\right)-\sum \lambda_{\alpha} Z\left(X_{\alpha}\right)\right]=0 \\
\text { So } \quad \sum_{\alpha=1}^{N} \lambda_{\alpha}=1
\end{gathered}
$$

An important parameter to evaluate estimation quality is that of considering estimation variance, defined as

$$
\sigma_{s}^{2}=2 \sum_{\alpha} \lambda_{\alpha} \gamma\left(x_{\alpha}-x_{0}\right)-\sum_{\alpha} \sum_{\beta} \lambda_{\alpha} \lambda_{\beta} \gamma\left(x_{\alpha}-x_{\beta}\right) .
$$

In order to obtain the best estimation, it will be necessary to minimize the estimation variance so:

$$
\partial \sigma^{2} / \partial \lambda_{\alpha}=0 \quad \forall \alpha=1, n
$$

With the exactness condition the system becomes:

$$
\left\{\begin{array}{c}
\sum_{\beta} \lambda_{\beta} \gamma_{\alpha \beta}+\mu=\gamma \\
\sum_{\beta} \lambda_{\beta}=1
\end{array} \quad \forall \alpha=1, n\right.
$$

The associate estimator is called Kriging and the system is called Kriging system. The coefficient's matrix does not depend on entity to be estimated but they are exclusively dependent on the sampled points position and on variografic function.

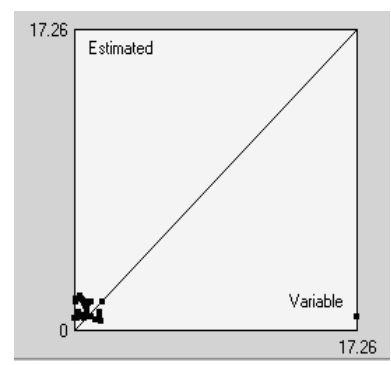

\begin{tabular}{|l|l|}
\hline \multicolumn{1}{|c|}{ Errors } & \multicolumn{1}{c|}{ Values } \\
\hline Mean error: & -0.1316 \\
Mean square error: & 8.358 \\
Sqrt( mean square error ]: & 2.891 \\
\hline Mean standard error: & -0.04896 \\
Mean square std error: & 1.332 \\
Sqrt! mean sqr std error ]: & 1.154 \\
\hline Mean estimation variance: & 5.786 \\
\hline Sqrt( mean estim. var.]: & 2.405 \\
\hline
\end{tabular}

Figure 7: $\quad$ Scatterplot and error table. 


\subsection{Cross validation}

The model has been tested using a cross validation method called "leave one out". This methodology estimates the variable's value in the known points and provides to delete from time to time the measured value. The error's report is shown below and geostatistical analysis is then completed according with the evaluation of scatterplot estimated/sampled values.

\subsection{Multivariate approach}

Co Kriging is an interpolation technique that allows one to use a more intensely sampled covariate in the estimation of values for a related variate. Because of the primary variate $\left(\mathrm{L}_{\mathrm{EQ}(\mathrm{A})}\right)$ correlation with a more available covariate $\left(\mathrm{L}_{\mathrm{P}}\right.$ at different emission frequencies), the Co Kriging technique is developed in order to improve interpolation estimates.

Let $\mathrm{Z}_{\mathrm{i} 0}\left(\mathrm{x}_{0}\right)$ be the value to estimated, the Co Kriging estimator $\mathrm{Z}_{\mathrm{i} 0}{ }^{*}\left(\mathrm{x}_{0}\right)$ becomes

$$
Z_{i 0}^{*}\left(x_{0}\right)=\sum_{i=1}^{N V} \sum_{\alpha=1}^{n_{i}} \lambda_{\alpha}^{i} z_{i}\left(x_{\alpha}\right)
$$

The set of weights $\lambda_{\alpha}^{i}$ can be defined according to the following two conditions: Correctness condition

$$
E\left[Z_{i 0}^{*}\left(x_{0}\right)\right]=E\left[z_{i 0}\left(x_{0}\right)\right]
$$

that becomes

$$
m_{i 0} \sum_{\alpha=1}^{n_{i o}} \lambda_{\alpha}^{i 0}+\sum_{i \neq i_{0}} m_{i} \sum_{\alpha=1}^{n_{i}} \lambda_{\alpha}^{i}=m_{i 0}
$$

This equation is satisfied by the following conditions

$$
\sum_{\alpha=1}^{n_{i 0}} \lambda_{\alpha}^{i 0}=1 \text { and } \sum_{\alpha=1}^{n_{i}} \lambda_{\alpha}^{i}=0
$$

that together with the condition of minimizing estimation function

$$
\sum_{j=1}^{N V} \sum_{\alpha=1}^{n_{i}} \lambda_{\alpha}^{i} \gamma_{i, j}\left(x_{k}-x_{\alpha}\right)+\mu_{i}=\gamma_{i i_{0}}\left(x_{k}-x_{0}\right)
$$

constitute the Co Kriging system.

\subsection{Variogram and regionalization model}

On experimental evidence it is developed a model based on one structure:

$>$ Spherical component

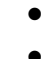

Sill 5,25

Range 40 


\subsection{Estimation map}
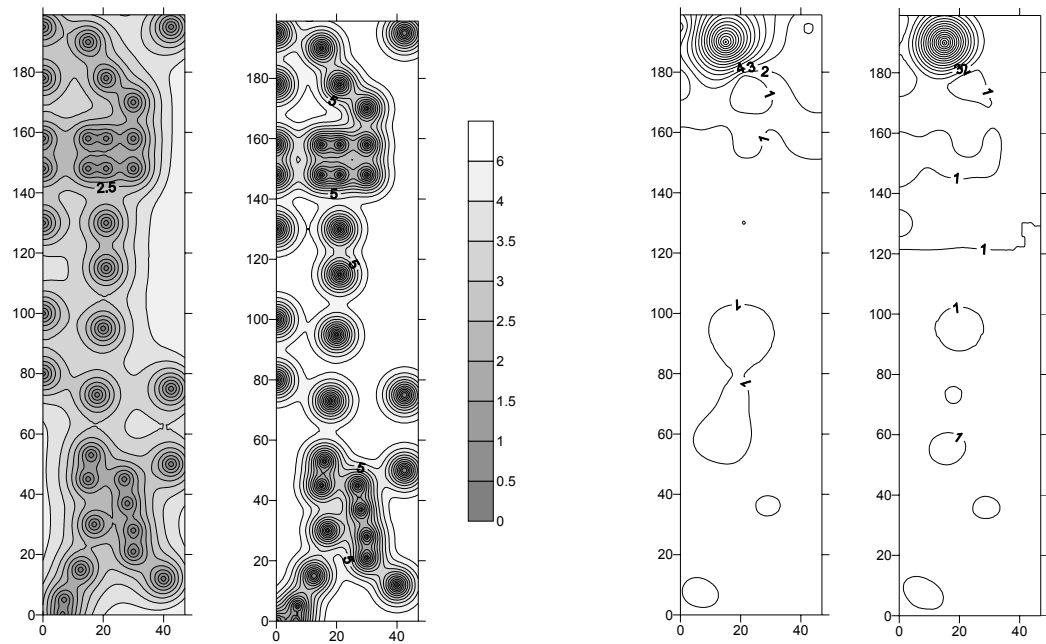

Figure 8: Estimation variance Map: Figure 9: Concentration Map: Co Co Kriging (left)/ Kriging (right). Kriging (left)/ Kriging (right).

\subsection{Cross validation}

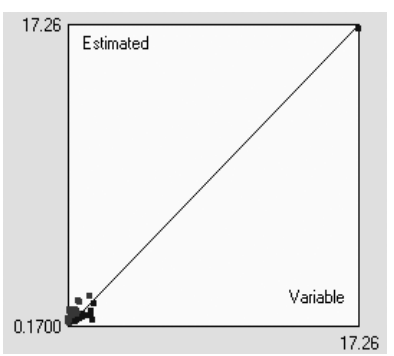

\begin{tabular}{|l|l|}
\hline \multicolumn{1}{|c|}{ Errors } & \multicolumn{1}{c|}{ Values } \\
\hline Mean error: & 0.01036 \\
Mean square error: & 0.1939 \\
Sqrt( mean square error): & 0.4404 \\
\hline Mean standard error: & -0.08708 \\
Mean square std error: & 1.004 \\
\hline Sqrt( mean sqr std error): & 1.002 \\
\hline Mean estimation variance: & 0.4753 \\
Sqrt( mean estim. var.): & 0.6894 \\
\hline
\end{tabular}

Figure 10: Scatterplot.

\section{Testing both models}

The final step is represented by the comparison between the ISC3 and the geostatistical approaches. These two sets of dust concentration values are tested according to the choice of six receptors in which samples are compared with the two model results. Two contour maps of the PM10 concentrations surrounding the quarry are shown. Data comparison shows the over prediction of PM 10 concentration by the ISC 3 model (on the right). This result may occur because the model applies the total emission of mobile sources to a specific area source. 
This application creates a constant uniform distribution of emissions over this area that does not correspond to the real case.

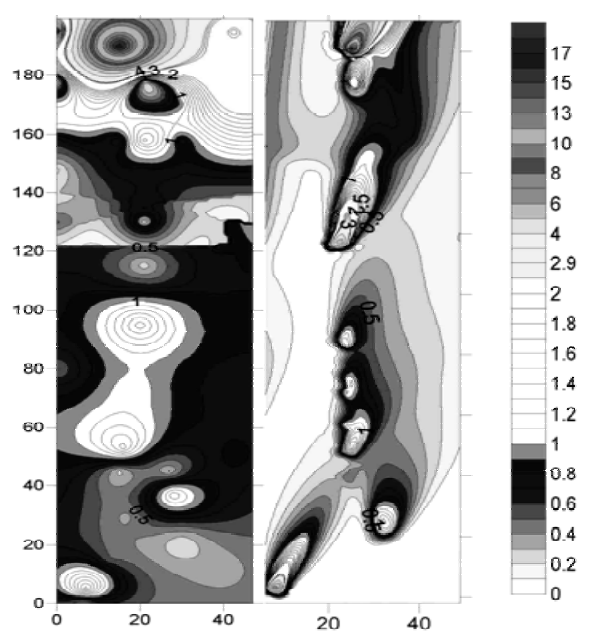

Figure 11: Comparison between estimation (left) and ISCST output (right).

\section{References}

[1] A geostatistic approach to the Functional Analysis Space Technique: a case of study". Alfaro Degan, Lippiello, Pinzari. ESREL 2005, Gdansk, 27 Giugno 2005.

[2] "Exposure to airborne dusts in the quarrying industry in Lazio". D. Lega et al. Prevenzione oggi. Quarterly review of studies and research on safety. ISPESL. April /June 2005.

[3] "Dust propagation: a method in risk analysis". Alfaro Degan G., Lippiello D., Pinzari $M$. Atti del 3rd Safety and Reliability International Conference -Konbin 2003- (26-30 May 2003; Gdynia, Poland).

[4] “Dispense di geostatistica applicata." Raspa G Università degli studi La Sapienza Dipartimento di Georisorse. 